\title{
Dynamic Facial Expression Recognition Using Local Patch and LBP-TOP
}

\author{
Yiming Wang, Hui Yu*, Brett Stevens, Honghai Liu \\ University of Portsmouth, Portsmouth, United Kingdom \\ *hui.yu@port.ac.uk
}

\begin{abstract}
Local binary pattern on three orthogonal planes (LBP-TOP) is one of the most popular method for dynamic texture analysis and has been successfully applied to facial expression analysis. Yet an effective LBP-TOP operator highly relies on preprocessing. And, like many appearance-based approaches, this approach reserves more identity-related cues rather than expression. In this work, we propose a fully automatic approach for facial expression recognition based on points registration, localized patch extraction and LBP-TOP feature representation. The efficiency of this method is evaluated on $\mathrm{CK}+$ database. Results show that the proposed method has achieved a better performance compared with existing methods.
\end{abstract}

\section{INTRODUCTION}

Automatic facial expression recognition has attracted increasing attention due to its various applications, such as computer technology, psychology and security among ohers. In this domain, dynamic analysis of facial expression is one of the most challenging problem because of the unpredictability of spatial and temporal extent. Facial expression dynamics are essential for facial behavior understanding since they describe basic facial muscle movements. Compared to frameby-frame spacial representations, spatial-temporal representations are considered to be more efficient and sensitive when representing subtle expressions [1]. They enable modelling temporal variance to capture higher level muscular activities and discriminate expressions that look similar in space. Much progress has been made to model spatial-temporal relations from facial image sequences [2], [3], [4], [5].

It is generally accepted that automatic machine recognition of facial expression include three subprocedures: (1) face registration, (2) feature extraction and representation, (3) facial feature analysis and expression recognition. Face registration aim at finding the region of interest, mostly the whole face, from image. Rigid registration perform the whole face alignment that align an input face to a prototypic frontal face. Another two registration methods are part registration (e.g. eyes, nose, mouth) [6] and points registration (fiducial points detection) [4]. Deriving an effective facial feature representation is the crucial step for facial expression analysis. Depending on the feature extraction process, facial representations can be divided into geometric-based approaches [4], [7] and appearance-based approaches [2], [5]. Geometricbased approaches present the motion of shape, especially facial points movements, while appearance-based approaches describe changes in skin texture, and it has been reported that the former ones often outperform the latter ones. The problem of facial feature analysis and expression recognition can be viewed as a classification problem. Classifiers, such as Support Vector Machine (SVM), Linear Discriminant Analysis (LDA), are employed to distinguish facial-expression-interpretative categories. The best known interpretative concepts of facial expressions include Action Units (AUs) recognition [8] and six basic emotions recognition (happy, sad, surprise, fear, angry, disgust).

Local binary pattern (LBP) is originally used to describe static local texture structures [9], and has shown advantages in the tolerance against illumination and its computational simplicity. Zhao et al. applied LBP feature extraction method on three orthogonal planes (LBP-TOP), which successfully introduced temporal relations to spatial LBP features [2]. They validated LBP-TOP on facial expression recognition as well as dynamic texture analysis, and results showed that LBP-TOP features outperformed spatial LBP features in the application to facial expressions. However, key issues existing in LBPTOP include challenging face registration and identity bias. Face registration is challenging because LBP-TOP need each frame in an image sequence to be in same size, or at least the subregions of each frame to be in same size. Any in-plane or out-plane rotation will degrade its performance. An effective LBP-TOP operator is highly dependent on face registration. The problem of identity bias generally exists in low-level features [1]. it means that the extracted features reserve more information about identity rather than expressions. Shan et al. proposed an person-independent approach using LBP [10], which highly relies on face registration. And it has not yet applied to facial expression dynamics.

Aiming at the these problems of LBP-TOP, we propose an approach to automatic recognize six basic emotions using local patch extraction and LBP-TOP representation. First, we detect point-based facial landmark by means of Supervised Descent Method (SDM) [11] which separately detects facial fiducial points in the first frame and tracks them in the following frames. Then, we extract local patches according to fiducial points. This extraction method has two main advantages: (a) the effect of identity bias can be better mitigate since the regions around fiducial points preserve more expressionrelated cues, and (b) within all the frames in a sequence, the location of subjects (e.g. eyes, nose) are more stable and facial texture movements are more smooth. In each patch of sequence, block-based approach is exploited where LBP-TOP features are extracted in each block and connected to represent 


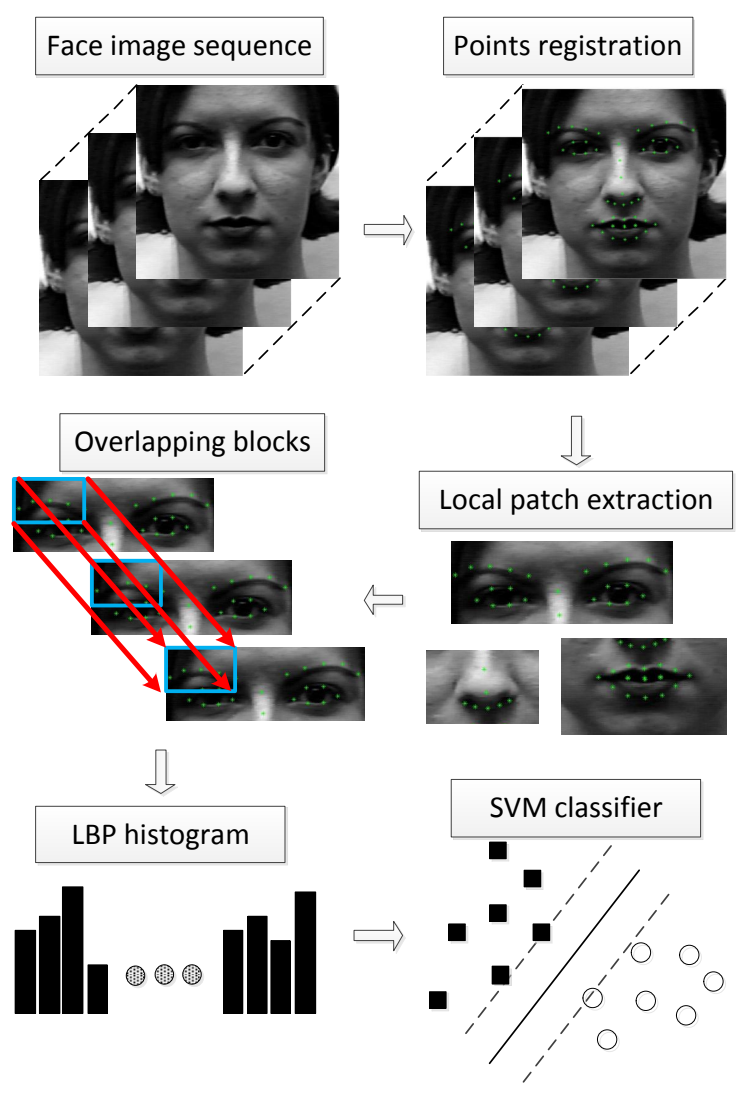

Fig. 1. The outline of proposed system

facial motions. Finally, we perform SVM classifier for emotion classification. The overview of this system is presented in Fig. 1.

The main contributions of this work include: (1) propose a novel method for emotion-enhanced feature extraction, (2) integrate the most effective and latest methods, such as SDM and LBP-TOP, for facial registration and facial representation. (3) the experimental results shows a good performance on person-independent facial expression recognition. The rest of the paper is organized as follows. Section II introduce the relevant works on dynamic analysis of facial expression. Theoretical components are described in III. The description of data sets and experimental evaluation are given in section IV. V conclude the paper with a summary and discussion.

\section{RELATED WORKS}

Approaches that only use geometric features mostly rely on facial fiducial points [4], [7], [12]. Geometric-based recognition can be viewed as facial points movement classification. Spatial relations and motions of these points are often used for expression analysis. In dynamic shape analysis, one can model the temporal characteristics into sequences of temporal segmentations: neutral, onset, apex and offset, which naturally solves the problem of unpredictability in temporal extent. Also, much research focus on pose-wise facial expression recognition [12], [13] and have made great contributions.

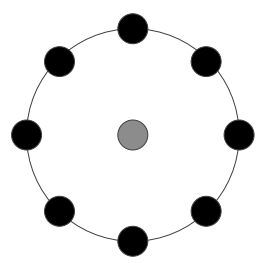

(a) Spot

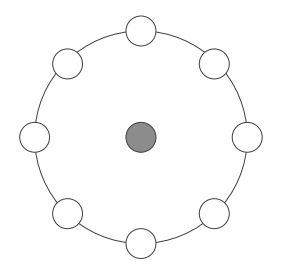

(b) Spot/flat

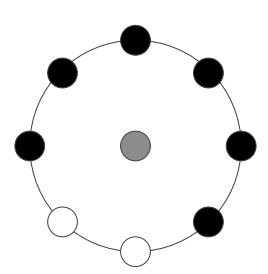

(c) Line end

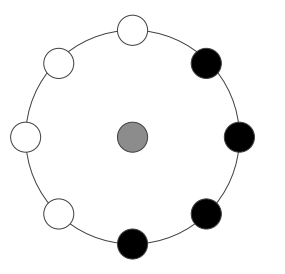

(d) Edge

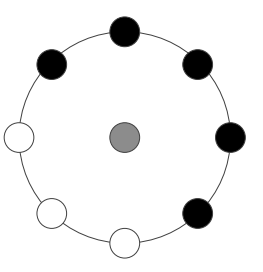

(e) Corner
Fig. 2. Examples of some uniform patterns that show meaningful local structures

However, these methods totally ignore the information presented in skin texture changes, and it is reported that they are often outperformed by appearance-based approaches. So in this work, we only focus on appearance-based methods.

Most appearance-based approaches applied low-level histogram representations. Popular low-level features include LBP, Local Phase Quantisation (LPQ) [14], Histogram of Gradients (HoG) [15], SIFT etc. Among them LBP and LPQ features extraction methods are extended to Three Orthogonal Plane for dynamic application.

LBP is one of the most commonly used method because it is computational simpler, powerful to present local structures and easily extended to its temporal model [16]. The original LBP operator encodes local texture variation with an integer which is derived by comparing each pixel with its 8 neighbors in $3 \times 3$ neighborhood. LBP histogram simply count these integers, and therefore deriving a 256-dim histogram representation. Ojala et al. described the extended LBP using circular neighborhoods which allows any radius and numbers of pixels, and showed a more discriminant representation with 59-element subset in which only those meaningful patterns (uniform pattern), such as spot, edge, corner shown in Fig. 2, are concerned [9]. Typically, we denote a LBP feature representation as $L B P_{(P, R)}^{u 2}$, where the notation $u 2$ stands for using uniform patterns only and $(P, R)$ defines a circular neighborhood of $P$ sampling points on the radius of $R$.

LBP-TOP extend spatial LBP features to the spatio-temporal domain. The regular LBP features (using circular neighborhood) are extracted from local spatio-temporal neighborhoods over three orthogonal planes: spatial plane XY, horizontal spatio-temporal plane XT and vertical sptio-temporal plane YT. Uniform patterns still constitute dominant components in representing temporal co-occurrences, so it is appropriate to use uniform patterns in three planes. It is reported that LBPTOP outperform its spatial counterpart in both emotion and AU recognition [1].

Considering the co-occurrences in subregions and their 

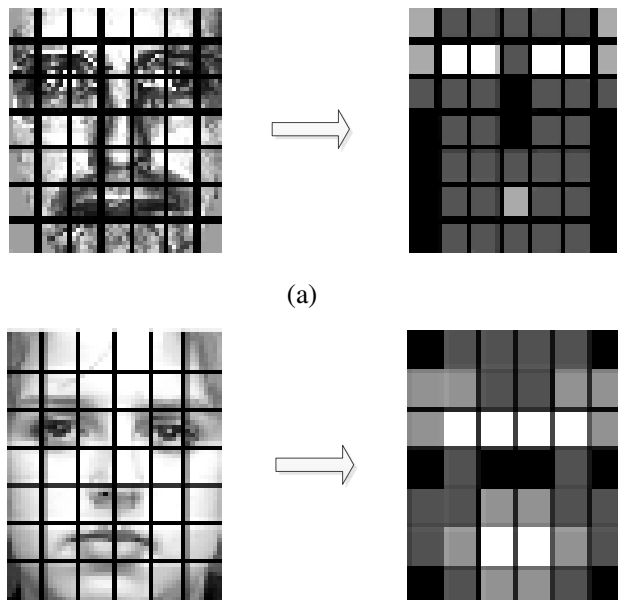

(a)

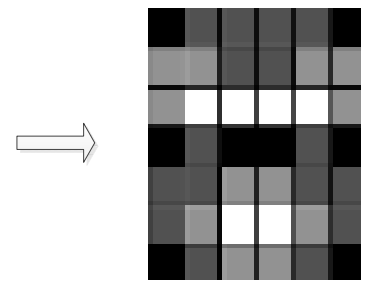

(b)

Fig. 3. Black squares indicate weight 0.0 , dark gray 1.0 , light gray 2.0 , and white 4.0. (a) Subregions were weighted in terms of identity variance. (b) The blocks were weighted according to expressions variance.

locations, recent research on LBP often use block-based technique for texture analysis [17], [18], [8]. This approach was first applied by Ahonen et al. in the application to face recognition [19]. They divided an face image into several nonoverlapping regions from which LBP features were extracted and concatenated into a spatially enhanced histogram. Considering that some certain regions contribute more than others regarding identity variance, the regions were correspondingly weighted based on their contributions. The weighted Chi square distribution was employed for this application. Zhao et al. extended this method to overlapping block-based approach in their experiments [2]. In the dynamic application to facial expression, best results was obtained with an overlapping ratio of $70 \%$ of original blocks. However, they did not apply any techniques to remove personal-related information. The deriving LBP descriptors were highly effected by identity bias. Shan et al. was also inspired by [19], they exploited an expression-based region weighted method that only considered the importance of expressions instead of identities [10]. Nevertheless, this method does not take advantage of overlapping blocks. The strict consistency of representative region make it difficult for face registration. Some works were done manually in preprocessing. Also, the experiment that was used to test person-independency did not achieve an ideal result.

\section{METHOD}

Our proposed method consist of three fundamental components. First, we localize the facial landmarks (49 fiducial points) using SDM. The detail of this technique is described in Section II-A. With the position of detected landmarks, we then extract local patches around fiducial points and apply LBPTOP in each patch (Section II-B). Finally, AdaBoost based feature selection technique and SVM classifier are briefly reviewed in Section II-C.

\section{A. Fiducial Landmark Detection}

In our study, We use SDM as facial landmark detection method. SDM is a non-parametric shape model for face alignment. Given an image with manually labeled landmarks which are referred to as $x_{*}$, face alignment can be defined as minimizing a Non-linear Least Square (NLS) function:

$$
f\left(x_{0}+\Delta x\right)=\left\|h\left(d\left(x_{0}+\Delta x\right)\right)-\phi_{*}\right\|_{2}^{2}
$$

where $\phi_{*}=h\left(d\left(x_{*}\right)\right)$ represents SIFT values and $x_{0}$ is initial configuration of landmarks. During training, SDM learns a sequence of generic descent directions. For testing image, it minimizes NLS objective function using learned decent directions.

In practical, a two-step preprocessing need to be done first: (1) detect a face using openCV face detector, and (2) initialize shape estimation by centering the mean face at normalized square. Then SDM can be execute to detect facial landmarks. Sometimes, one need to perform Principle Component Analysis (PCA) for dimensionality reduction. For tracking task, SDM perform detection in each frame by initializing the landmark estimation from its previous frame. We employed this method in this work because it is extremely fast and accurate. points registration which detect fiducial points in the first frame of image sequence and track them in the rest.

The interested reader can refer to [11] for more details of SDM. The $\mathrm{C} / \mathrm{C}++$ and MATLAB implementations of SDM (the model has been trained already) can be found online [20].

\section{B. Extract Localized Patches}

Before this step, a preprocess is need where eyes positions are used for alignment in case of in-plane rotation, as is done by most papers [2], [8], [5], [17]. Considering the importance of expression, we extract three local patches around nose, mouth and eyes regions that are considered to make more contribution to expression variance. Following the registration of facial landmark points, local patches are extracted based on the position of these points. There are some certain points which are always in a fixed position regardless of any changes of facial muscles. Depending on these points, a localized texture patch is obtained by establishing a point-centered bounding box with a fixed size in each frame. An image sequence is therefore broken down into its fundamental patch sequences. In this experiment, three patches were extracted from the regions corresponding to eyes, nose and mouth, respectively, as is shown in Fig.4. In each patch, we perform block-based technique to capture local facial motions. We chose $2 \times 8$ blocks in eyes-related patch, $2 \times 2$ for noserelated patch and $5 \times 7$ for mouth-related patch. According to the research in [2], the overlapping ratio $r a^{\prime}$ in height is computed as followed:

$$
r a^{\prime}=\frac{r a \cdot h / r}{\frac{(r a \cdot h \cdot(r-1)) / r+h}{r}}=\frac{r a \cdot r}{r a \cdot(r-1)+r}
$$

where $r a$ is original overlapping ratio, $h$ is the hight of block, and $r$ is row number of blocks. 


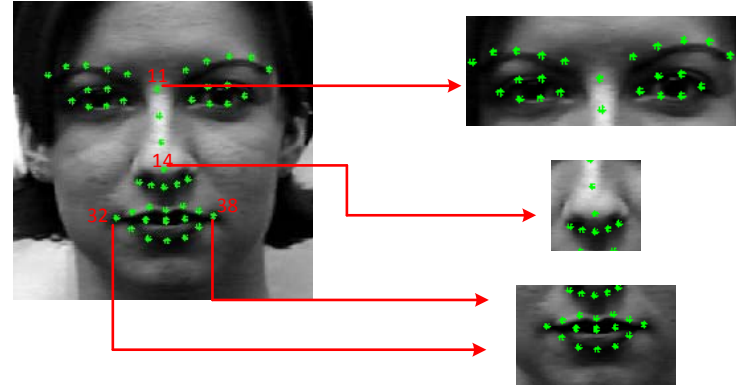

Fig. 4. Local patch extraction: the first patch centers around point number 11, the second centers around point number 14, point number 32 and 38 are used to design the bounding box of the third patch.

The LBP-TOP histogram is computed over each block from each patch sequence. The final histogram concatenate all these histograms into a single vector to represent the whole image sequence. Empirically, we will introduce uniform patterns to our LBP-TOP operators, which results in a histogram representation of 177 bins per sequence. The corresponding

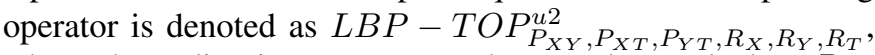
where the radius in axes $\mathrm{X}, \mathrm{Y}$ and $\mathrm{T}$ can be marked as $R_{X}$, $R_{Y}$ and $R_{T}$, and $P_{X Y}, P_{X T}$ and $P_{Y T}$ represent the number of neighborhood points in $X Y, X T$ and $Y T$.

\section{Support Vector Machine}

SVM is considered as one of the most robust and accurate method for data classification. It provides a good balance between model complexity and generalization error. In a twoclass learning task, SVM finds a hyperplane with maximal margin for linear separable data. In a geometrical view, this approach ensures both the accurate partition in training data and the best generalization ability on test data. For nonlinear data, SVM allows domain-specific kernel functions that map the input data to a different space of larger dimensions. The most frequently used kernel functions are polynomial and Radial Basis Function (RBF) kernels.

SVM only makes binary decisions. For multi-class problem, a simple but effective technique is one-vs-rest approach. It trains the binary classifiers via distinguish one class against all the others. Each class trains a corresponding binary classifier. The final decision for a test case is made according to the the geometrical distance between test point and decision boundary of all the binary classifiers (the classifier with the largest distance is chosen).

\section{EXPERIMENT}

The efficiency of proposed method is test on CohnKanade Extended $(\mathrm{CK}+)$ database. The overall performance and person-independent performance will be test respectively.

\section{A. Dataset}

$\mathrm{CK}+$ is a posed facial expression database [21]. It consist of 210 adults whose age range from 18 to 50 . It contains 123 subjects and 593 frontal image sequence in which the facial expressions of subjects are displayed from neutral to target emotions. From these, only 327 sequences from 118 subjects fit the prototypical definition of emotions and therefore, are annotated with seven universal emotions (six basic and contempt). Within a image sequence,68 landmark points are manually annotated in all images, and action units and their intensity are also provided for the peak frame. In our study, 309 sequences that are explicitly labeled one of the six basic emotions are selected.

\section{B. Evaluation}

In this experiment, an overlapping ratio of $70 \%$ of these blocks is selected, which shows an outstanding performance in previous work [2]. Corresponding overlapping ratio in both height and width is about $43 \%$.

We did the experiments using uniform patterns with a neighborhood of $P=(8,8,8)$ and radii of $R=(3,3,3)$, denoted as $L B P-T O P_{8,8,8,3,3,3}^{u 2}$. For evaluation, we trained SVM classifier with RBF kernel function using "leave one subject out" cross-validation.

As we can see in Table 1, happy, surprise and disgust are recognized with high accuracy $(100 \%, 97.44 \%$ and $100 \%$, each), whereas the recognition rates of the others are relatively low. Table 2 presents the comparison of different methods. [4] is geometric-based approach, whilst the others are appearancebased approaches. It is clear that appearance-based methods outperform shape model. In this stage, the proposed method does not perform so good as the other two appearance-based approaches. Unlike the whole face representation, our method achieves a partial face representation, which will cause the loss of information, as well as the reduction of accuracy. In the real-world applications, we believe it is reasonable to improve person-independent emotion recognition that partially sacrifices the accuracy.

In former experiments, person-independent cases are not emphasizes. Nearly every testing face (subject) could appear in training sets. In order to evaluate person-independent performance, we randomly choose half of the 118 subjects (individuals) for training and the other half for testing, associated emotion sequences are also divided. This scheme guarantees that the subjects used for training will never appear in testing. The testing on a totally new faces is therefore person-independent. This step is repeated ten times to achieve the average results.

As is described in table 3 , our method achieves the best performance on person-independent experiments. The other two method show a dramatic drop in person-independent performance. Meanwhile, our proposed method decrease very slightly (only 1\%).

\section{CONCLUSION}

In this work, we focus on the task of facial expression dynamics learning and person-independent emotion estimation using localized, block-based LBP-TOP feature representation. The main contribution in this study is that we proposed local patch extraction method that extracted local patches from 
TABLE I

CONFUSION MATRIX (\%) OF 6-CLASS FACIAL EXPRESSION RECOGNITION FOR THIS WORK

\begin{tabular}{|c|c|c|c|c|c|c|}
\hline & Angry & Disgust & Fear & Happy & Sadness & Surprise \\
\hline Angry & $\mathbf{7 5 . 0 0}$ & 4.17 & 0 & 20.83 & 0 & 0 \\
\hline Disgust & 0 & $\mathbf{1 0 0 . 0 0}$ & 0 & 0 & 0 & 0 \\
\hline Fear & 0 & 0 & $\mathbf{5 7 . 1 4}$ & 28.57 & 0 & 14.29 \\
\hline Happy & 0 & 0 & 0 & $\mathbf{1 0 0 . 0 0}$ & 0 & 0 \\
\hline Sadness & 16.67 & 0 & 0 & 16.67 & $\mathbf{6 6 . 6 7}$ & 0 \\
\hline Surprise & 0 & 0 & 0 & 2.56 & 0 & $\mathbf{9 7 . 4 4}$ \\
\hline
\end{tabular}

TABLE II

COMPARISON OF DIFFERENT METHOD FOR “LEAVE ONE SUBJECT OUT" CROSS-VALIDATION

\begin{tabular}{|c|c|c|c|c|c|c|c|}
\hline & Angry & Disgust & Fear & Happy & Sadness & Surprise & Total \\
\hline$[2]$ & 86.67 & 100.00 & 92.00 & 100.00 & 90.91 & 98.80 & 94.50 \\
\hline$[4]$ & 91.1 & 94.0 & 83.3 & 89.8 & 76.0 & 91.3 & 86.3 \\
\hline$[10]$ & 86.67 & 96.61 & 84.00 & 100.00 & 67.86 & 98.80 & 92.88 \\
\hline Ours & 75.00 & 100.00 & 57.14 & 100.00 & 66.67 & 97.44 & 87.74 \\
\hline
\end{tabular}

TABLE III

COMPARISON OF DIFFERENT METHOD FOR "LEAVE ONE SUBJECT OUT" CROSS-VALIDATION AND PERSON-INDEPENDENT TEST

\begin{tabular}{|c|c|c|}
\hline & Cross-validation & Person-independent \\
\hline$[2]$ & 92.88 & 85.16 \\
\hline$[10]$ & 94.50 & 85.89 \\
\hline Ours & 87.74 & 86.56 \\
\hline
\end{tabular}

fiducial point-centered, fixed-sized bounding boxes and preserve information about their spatial relations. These patches are considered more important for human beings to perform expressions. Thus, a person-independent, spatially enhanced feature representation is obtained.

Experiments on $\mathrm{CK}+$ database with person-independent implementation show that our method is effective and favorable compared to other methods. Our approach is more flexible for real application problems, because it not only achieves a good solution to identity bias, but also allows tolerance against small changes in the facial image size and head-pose. In future, we would like to combine geometric-based approaches to normalize head-pose variation and temporal extent.

\section{REFERENCES}

[1] E. Sariyanidi, H. Gunes, and A. Cavallaro, "Automatic analysis of facial affect: A survey of registration, representation and recognition," IEEE Transaction on Pattern Analysis and Machine Intelligence, vol. 99, 2014.

[2] G. Zhao and M. Pietikainen, "Dynamic texture recognition using local binary patterns with an application to facial expression," IEEE Transaction on Pattern Analysis and Machine Intelligence, vol. 29, no. 6, pp. 915-928, 2007.
[3] S. Koelstra, M. Pantic, and I. Patras, "A dynamic texture-based approach to recognition of facial actions and their temporal models," IEEE Transaction on Pattern Analysis and Machine Intelligence, vol. 32, no. 11, pp. 1940-1954, 2010.

[4] Z. Wang, S. Wang, and Q. Ji, "Capturing complex spatio-temporal relations among facial muscles for facial expression recognition," in IEEE Conference on Computer Vision and Pattern Recognition, pp. 34223429, 2013.

[5] B. Jiang, M. Valstar, B. Martinez, and M. Pantic, "A dynamic appearance descriptor approach to facial actions temporal modelling," IEEE Transaction on Cybernetics, vol. 44, no. 2, pp. 161-174, 2014.

[6] L. Jeni, J. Girard, J. Cohn, and F. D. L. Torre, "Continuous au intensity estimation using localized, sparse facial feature sapce," in 10th IEEE International Conference and Workshops on Automatic Face and Gesture Recognition, pp. 1-7, 2013.

[7] M. Valstar and M. Pantic, "Fully automatic recognition of the temporal phases of facial actions," IEEE Transactions on Systems, Man, and Cybernetics, Part B: Cybernetics, vol. 42, no. 1, pp. 28-43, 2012.

[8] S. Taheri, Q. Qiu, and R. Chellappa, "Structure-preserving sparse decomposition for facial expression analysis," IEEE Transactions on Image Processing, vol. 23, no. 8, pp. 3590-3603, 2014.

[9] T. Ojala, M. Pietikainen, and T. Maenpaa, "Multiresolution gray-scale and rotation invariant texture classification with local binary patterns," IEEE Transaction on Pattern Analysis and Machine Intelligence, vol. 24, no. 7, pp. 971-987, 2002.

[10] C. Shan, S. Gong, and P. McOwan, "Facial expression recognition based on local binary patterns: A comprehensive study," Image and Vision Computing, vol. 27, no. 6, pp. 803-816, 2009. 
[11] X. Xiong and F. D. la Torre, "Supervised descent method and its applications to face alignment," in IEEE Conference on Computer Vision and Pattern Recognition, pp. 532-539, 2013.

[12] O. Rudovic, M. Pantic, and I. Patras, "Coupled gaussian processes for pose-invariant facial expression recognition," IEEE Transaction on Pattern Analysis and Machine Intelligence, vol. 35, no. 6, pp. 13571369, 2013.

[13] Y. Tong, J. Chen, and Q. Ji, "A unified probabilistic framework for spontaneous facial action modeling and understanding," IEEE Transaction on Pattern Analysis and Machine Intelligence, vol. 32, no. 2, pp. 258-273, 2010.

[14] V. Ojansivu and J. Heikkila, "Blur intensitive texture classification using local phase quantization," in Image and signal processing, pp. 236-243, 2008.

[15] N. Dalal and B. Triggs, "Histograms of oriented gradients for human detection," in IEEE Conference on Computer Vision and Pattern Recognition, vol. 1, pp. 886-893, 2005.

[16] D. Huang, C. Shan, M. Ardabilian, Y. Wang, and L. Chen, "Local binary patterns and its application to facial image analysis: A survey," IEEE Transactions on Systems, Man, and Cybernetics, Part C: Aplications and Reviews, vol. 41, no. 6, pp. 765-781, 2011.

[17] M. Valstar, B. Jiang, M. Mehu, M. Pantic, and K. Scherer, "The first facial expression recognition and analysis challenge," in IEEE International Conference and Workshops on Automatic Face and Gesture Recognition, pp. 921-926, 2011.

[18] B. Jiang, M. Valstar, and M. Pantic, "Action unit detection using sparse appearance descriptors in space-time video volumes," in IEEE International Conference and Workshops on Automatic Face and Gesture Recognition, pp. 314-321, 2011.

[19] T. Ahonen and M. Pietikainen, "Face description with local binary patterns: application to face recognition," IEEE Transaction on Pattern Analysis and Machine Intelligence, vol. 28, no. 12, pp. 2037-2041, 2006.

[20] [online]. Available: http://www.humansensing.cs.cmu.edu/intraface.

[21] P. Lucey, J. Cohn, T. Kanade, J. Saragih, Z. Ambadar, and I. Matthews, "The extended cohn-kanade dataset (ck+): A complete dataset for action unit and emotion specified expression," in IEEE Computer Society Conference on Computer Vision and Pattern Recognition Workshops, pp. 94-101, 2010. 